\title{
émulations
}

\section{La maison des devises, un capital transnational}

\section{Les investissements immobiliers des migrants équatoriens en vue du retour, à la lumière de la crise économique en Espagne}

\author{
Anna Perraudin \\ Émulations - Revue de sciences sociales \\ 2020, n³4, «Transnationaliser le retour. Vers une révision du regard sur les \\ migrations de retour contemporaines ».
}

\section{Article disponible à l'adresse suivante}

https://ojs.uclouvain.be/index.php/emulations/article/view/perraudin

\section{Pour citer cet article}

Anna Perraudin, « La maison des devises, un capital transnational. Les investissements immobiliers des migrants équatoriens en vue du retour, à la lumière de la crise économique en Espagne », Émulations, n 34, Mise en ligne le 7 octobre 2020. DOI : 10.14428/emulations.034.06

Distribution électronique : Université catholique de Louvain (Belgique) : ojs.uclouvain.be

(C) Cet article est mis à disposition selon les termes de la Licence Creative Commons Attribution, Pas d'Utilisation Commerciale 4.0 International. http://creativecommons.org/licenses/by-nc/4.0/

Éditeur : Émulations - Revue de sciences sociales / Presses universitaires de Louvain https://ojs.uclouvain.be/index.php/emulations

ISSN électronique : $1784-5734$

$\frac{\text { PUL PRESSES }}{\text { UNIVERSITAIRES }}$




\title{
La maison des devises, un capital transnational
}

\author{
Les investissements immobiliers des migrants \\ équatoriens en vue du retour, à la lumière de la crise \\ économique en Espagne
}

Anna Perraudin ${ }^{1}$

\begin{abstract}
[Résumé] À partir du cas de l'Espagne, remarquable en raison du mouvement des retours au pays chez les immigrés autour de 2010, attribués à la crise économique, l'article discute l'influence des maisons achetées dans les lieux d'origine sur les trajectoires de retour. L'analyse s'appuie sur une enquête qualitative réalisée entre 2015 et 2018 à Quito et Madrid (entretiens, observations auprès de seize ménages). L'ancrage de l'observation dans les villes donne à voir une autre dimension des maisons de retour que celle qui domine dans la littérature classique : fondues dans le tissu urbain, elles composent un patrimoine économique, générant une rente locative. Centrales dans les projets migratoires de nombreux migrants aux revenus modestes, les « maisons des devises » seront considérées comme des capitaux investis, qui peuvent circuler ou être convertis en d'autres ressources, selon l'éclairage de la théorie de l'accumulation des capitaux. Ces biens immobiliers opèrent donc comme des capitaux transnationaux, fruits de stratégies familiales : ils peuvent permettre une réinstallation dans le pays d'origine, mais aussi paradoxalement prolonger le séjour en Espagne.
\end{abstract}

Mots-clés : migrations de retours, capitaux transnationaux, logement, Espagne, Équateur.

Remittance houses, a transnational asset. Ecuadorian migrants' transnational investments in housing, from the perspective of the Spanish economic crisis

[Abstract] Based on the case of Spain, remarkable for the relocation of immigrants returning to their home country around 2010, the article discusses the significance of houses purchased in places of origin and their impact on the decision to return to the home country. The analysis is based on a qualitative survey conducted between 2015 and 2018 in Quito and Madrid (interviews, observations in 16 households). Conducting the survey in cities reveals another facet of remittance houses than that prevalent in classical literature. Embedded in the urban fabric, they constitute an economic heritage generating rental income. Essential to migration projects of many low-income migrants, "remittance houses" will be considered as invested assets that can circulate or be converted into other resources, according to asset accumulation theory. These properties thus act as transnational investments, the result of family strategies; they can enable resettlement in the country of origin, but also, paradoxically, extend the stay in Spain.

Keywords: return migration, transnational assets, housing, Spain, Ecuador.

\footnotetext{
${ }^{1}$ CNRS, Université de Tours, CITERES, France. Fellow de l'institut Convergences Migrations.
} 


\section{Introduction}

Images de familles attendant au milieu de montagnes de valises l'avion qui les ramènera au pays, pancartes de programmes d'aides au retour placardées dans le métro à Madrid... Mis en scène et amplifiés par les discours politiques et les médias, les départs des immigrés de l'Espagne après la crise économique de 2008 ne sont pas passés inaperçus. Il est vrai que " la crise », selon le raccourci en usage en Espagne, si elle n’a pas épargné les autochtones, s'est avérée particulièrement brutale pour la maind'œuvre étrangère qui avait afflué à la fin des années 1990, au moment du plein emploi. Le chômage, la dégradation des conditions de travail pour ceux qui en avaient encore, les politiques sociales d'austérité, l'effondrement du marché immobilier, la hausse des taux d'intérêt et l'endettement de ceux qui étaient parvenus à accéder à la propriété, le durcissement des politiques migratoires, ont multiplié les obstacles à la poursuite du projet migratoire. Dans ce contexte, les départs de l'Espagne ont été présentés par les médias comme une des expressions de l'intensité de la crise (Perraudin, 2016).

Le caractère contraint, soudain, attribué à ces mobilités ne doit toutefois pas faire oublier que le retour est souvent un point de mire du projet migratoire (Sayad, 1975). Il a pu être préparé par des investissements visant à obtenir une mobilité sociale dans le pays d'origine, réalisés bien avant que la réinstallation ne soit envisagée : maintien de lien avec le pays et les proches, épargne, construction d'une maison, autant d'entreprises facilitées par l'inscription du migrant dans un champ social transnational (Glick Schiller, 1999 ; Schramm, 2011). Même précipités, les départs peuvent alors s’appuyer sur des capitaux accumulés (Cortés, 2011). C’est dans cette temporalité du projet migratoire, entre la préparation du retour à moyen terme et sa mise en œuvre, que se situe la réflexion développée dans ce texte. Pour les migrants qui quittent l'Espagne en ayant préparé, en amont, leur retour, quels capitaux facilitent la réinstallation en Équateur? On s'intéressera ici aux maisons achetées par les migrants dans leur pays d’origine, souvent au cœur des projets migratoires. « Maisons de retour» (Pinson, 1999) ou « maisons des devises »-remittance houses, en anglais (López 2010), cette dernière expression renvoyant à la dimension économique de l'investissement plus qu'à la finalité du projet ${ }^{2}-$, comment font-elles ressource dans les projets de retour?

Le lien entre les investissements immobiliers réalisés en vue du retour et la réalisation de ce dernier sera interrogé à la lumière des (im)mobilités des Équatoriens ayant migré en Espagne avant 2008. Ce groupe national, qui constitue le second groupe immigré non européen après les Marocains depuis les années 2000, est en effet identifié comme une des principales populations étrangères étant retournées dans leur pays

\footnotetext{
${ }^{2}$ La notion de remittance houses, sans être une expression vernaculaire, n'est pas non plus très diffusée dans une littérature scientifique qui s'est de façon générale peu intéressée aux maisons des migrants. Sarah Lynn López la définit de la façon suivante : « a house built with money earned by a Mexican migrant in the United States who sends dollars - remits - to Mexico for the construction of his or her dream house. More broadly, I use this term to emphasize remitting and migration as key components of contemporary transnational building practices across the globe » (López, 2010 : 33). Les maisons qu'elle observe se situent dans le contexte traditionnel du monde rural mexicain.
} 
d'origine après la crise'. Paradoxalement, c'est aussi l'un des groupes qui avait le plus investi dans l'immobilier en Espagne et qui de ce fait y a été touché par la dette immobilière. Ceux qui sont rentrés en Équateur à la suite de la crise sont-ils ceux qui y avaient préparé leur retour ? Ceux qui restent en Espagne malgré les difficultés créées par la crise économique sont-ils ceux qui y avaient tout misé ? Comment et où les capitaux constitués par l'immobilier, accumulés en vue du retour, sont-ils mis à profit ?

Tout en mobilisant des données secondaires (statistiques et rapports sur les migrations équatoriennes en Espagne et les politiques publiques équatoriennes), des informations obtenues auprès de personnes-ressources (consulat équatorien à Madrid, Communauté de Madrid, etc.) et sur les sites internet d'agences immobilières, l'analyse se fonde sur des observations et des entretiens semi-directifs réalisés avec six migrants de retour en Équateur (terrain de dix jours en 2015 à Quito) et dix migrants étant restés en Espagne (trois terrains de quinze jours en 2014, 2016, 2018 à Madrid). Provenant de régions diverses en Équateur, ils sont arrivés en Espagne entre 1990 et 2003 et s'y inséraient dans les mêmes secteurs d'emploi, caractérisés par une nette division ethnique et genrée (service à la personne et restauration pour les femmes, secteur du bâtiment pour les hommes). Leurs revenus assez homogènes les inscrivent en Espagne dans la catégorie des ménages modestes. La démarche compréhensive permet de saisir la temporalité des investissements, leurs justifications, et les liens complexes, parfois contradictoires, entre projets de retour et investissements immobiliers.

L'article proposera d'abord un état des lieux des connaissances sur les retours attribués à la crise économique en Espagne, et l'hypothèse selon laquelle les maisons dans les pays d'origine relèvent d'un capital susceptible d'encourager les mobilités de retour sera discutée. Dans un second temps, l'enquête de terrain montrera comment ces maisons peuvent fonctionner comme un capital transnational. Dans les villes, où l'ensemble des enquêtés avaient préparé leur retour, une partie de ces biens génère une rente locative, et joue de ce fait un rôle important dans le projet migratoire bien avant un éventuel retour ${ }^{5}$. Enfin sera analysée la façon dont la crise économique vient bouleverser les projets migratoires, et amène à utiliser le patrimoine constitué par les migrants, selon une pluralité de scénarios.

\footnotetext{
${ }^{3}$ La migration équatorienne trouve son essor en Espagne entre la fin des années 1990 et le milieu des années 2000. L'Équateur traverse alors une grave crise politique et économique tandis que l'Espagne, en plein boom économique, recrute une main-d'œuvre peu qualifiée. Grâce à une politique migratoire favorable aux Latino-Américains, le regroupement des familles en Espagne est rapide.

${ }^{4}$ L'enquête de terrain a vocation à être poursuivie : ces résultats sont préliminaires.

${ }^{5}$ De par son inscription dans les études migratoires, cette étude ne prétend pas aborder toute la complexité du fonctionnement du marché immobilier, en Espagne ou en Équateur.
} 


\section{Qualifier les retours dans le contexte de la crise}

Alors que les retours ont longtemps été négligés dans la littérature sur les migrations, ceux attribués à la crise économique en Espagne ont été très étudiés ${ }^{6}$, surtout pour les Latino-Américains. Le contexte espagnol soulève en effet de nombreuses questions : l'Espagne est devenue en une dizaine d'années à peine, pendant l'embellie économique, un pays d'immigration de main-d'œuvre. Selon l'Instituto Nacional de Estadísticas, entre 1998 et 2008, la part des immigrés dans la population passe de 1,6 \% à 11,4 \%. Ces immigrés fraîchement installés, moteurs de l'économie espagnole et en particulier du secteur du bâtiment, sont encore plus touchés que les autochtones par le chômage après le retournement de conjoncture ${ }^{7}$. Une approche des migrations fondée sur la rationalité économique voudrait que, face à ces obstacles, les migrants quittent massivement l'Espagne. Or les enquêtes statistiques et qualitatives présentent un panorama bien plus nuancé. Certes, en 2011, le solde migratoire espagnol est négatif et les statistiques affichent une hausse des départs alimentée par les immigrés, mais dans des proportions très éloignées de l'hémorragie que laissaient entrevoir certaines prévisions. Ainsi, et bien qu'il soit difficile de quantifier le phénomène avec précision ${ }^{8}$, entre 65000 et 85000 personnes d'origine équatorienne ayant résidé en Espagne seraient retournées en Équateur, soit entre 13 et 17 \% de ce segment de la population (Moreno-Márquez, Álvarez-Román, 2017). Par ailleurs, les départs de l'Espagne sont loin d'être toujours des « retours » dans le pays d'origine : ils ont parfois comme destination d'autres pays européens (Belgique, Suisse, Allemagne, Royaume-Uni ${ }^{9}$ ). Les enquêtes qualitatives éclairent d'autres complexités : dans la famille, parents, enfants, mais aussi maris et femmes ne partent pas toujours au même moment et aux mêmes endroits, ce qui appelle à une lecture des « retours » attentive au genre et aux générations (Cortes, Oso, 2017; Ramos, 2017).

Qui sont alors ceux qui quittent l'Espagne pour leur pays d'origine ? Ce ne sont pas nécessairement les plus fragiles. Des enquêtes sur les stratégies migratoires des migrants boliviens en Espagne montrent que, même lorsque leurs conditions de vie et leurs revenus se sont dégradés, s'ils n'ont pas réussi à épargner, la plupart cherchent à éviter un retour dans le pays d'origine qui s'apparenterait à un échec (Baby-Collin, Cortes, 2014 ; Bastia 2011). De même, les migrants en situation irrégulière tendent à prolonger leur séjour en attendant une évolution de leur statut (Ibid.). Les Équatoriens

\footnotetext{
${ }^{6}$ Voir par exemple López de Lerra, 2010 ; Larramona, 2013 ; Bastia, 2011 ; Baby-Collin, Cortes, 2014 ; Parella, Petroff, 2014.

7 En 2010, le taux de chômage est de 18,4 \% pour les autochtones, 39,1 \% pour les immigrés (IOE, $2011: 89)$.

${ }^{8}$ Je ne reviendrai pas ici sur les méthodes de calcul des mobilités des immigrés et sur leurs limites (Perraudin, 2016).

${ }^{9}$ Ces schémas de mobilité s'expliquent par la politique migratoire de l'Espagne. Jusqu'au milieu des années 2000, les Latino-Américains y ont bénéficié de conditions préférentielles d'obtention de titres de séjour et de naturalisation, par rapport aux migrants d'autres origines : grâce à ces statuts administratifs, ils peuvent se déplacer et travailler dans l'espace européen.
} 
sont toutefois peu concernés par cette dernière stratégie, puisqu'en 2007 seuls 5,9 \% étaient en Espagne sans titre de séjour (Cerrutti et al., 2011 : 63).

Ces observations amènent à interroger le rôle de la crise dans ces mobilités. À partir de son expérience auprès des migrants, un agent du consulat équatorien à Madrid, rencontré en 2014, distingue trois types de retours : les retours préparés, lorsque le migrant considère avoir rempli ses objectifs initiaux et clôt son cycle migratoire de sa propre initiative ; les retours précipités, lorsque le retour avait été envisagé à plus long terme et est accéléré par la crise ; les « fuites », en particulier dans les cas de fort endettement, sans aucune préparation préalable. Le caractère planifié ou imprévu des retours apparaît alors comme une variable plus pertinente que les déterminants économiques pour distinguer les modalités de la réinstallation dans le pays d'origine (même si les deux peuvent être liés) (Cortés, 2011). Une enquête menée après 2008 aux États-Unis sur les « retours » des migrants relativise également le poids de la crise économique sur les mobilités, en montrant qu'outre les éléments conjoncturels liés au renversement économique (perte d'emploi, dégradation des conditions de travail, xénophobie accrue, etc.), jouent des éléments plus personnels (cycle de vie, localisation de la famille, etc.) ainsi que des facteurs externes, comme la possibilité de se projeter de façon positive dans le pays d'origine (Guarnizo, 2014). À noter sur ce point qu'au moment où l'économie espagnole sombrait, les indicateurs économiques dessinaient l'image d'une économie équatorienne redynamisée. Beaucoup d'attentes reposaient sur les changements annoncés par le gouvernement Correa (éducation, emploi, infrastructures), grâce à un travail actif, de la part de ce gouvernement, pour regagner la confiance des migrants (Koller, 2010).

La discussion du rôle de la « crise » dans la décision du retour et sa mise en œuvre invite donc à replacer le moment du retour dans une temporalité plus large et à en éclairer les déterminants multiples. Sans éluder cet aspect multidimensionnel, il est intéressant de porter l'attention sur les ressources qui permettent de construire ces trajectoires de mobilité. Les notions de « compétences migratoires » et de «capital de mobilité » se réfèrent à des aptitudes et des ressources, matérielles et immatérielles, en partie acquises au fil de la « carrière migratoire », qui permettent la mobilité géographique et l'orientent (Ma Mung, 2009; Martiniello, Rea, 2011 ; Tarrius, 2001). Dans une optique complémentaire, la théorie de « l'accumulation des capitaux transnationaux », issue d'un débat sur la mobilité sociale, développe l'idée stimulante que ces ressources peuvent (ou non) être accumulées, investies et transférées d'un contexte à l'autre (Cortés, 2011 ; Suárez-Grimalt, 2017). Les travaux inspirés par cette réflexion ont pour l'heure surtout porté sur le marché du travail. Ils montrent les limites des préparatifs préalables au retour et la difficulté à convertir les capitaux immatériels acquis pendant la migration : les migrants, surtout les femmes, peinent à valoriser en Équateur les compétences développées en Espagne (Herrera, Pérez Martínez, 2015 ; Martínez Buján, 2016 ; Vega, 2016). Qu'en est-il pour les préparatifs relatifs au logement? 
Bien qu'ancrées dans un territoire, les maisons des migrants représentent un capital transnational. Elles sont un patrimoine matériel, constitué pour soi ou pour les siens, souvent au prix d'efforts conséquents. De nombreux ménages immigrés y consacrent une partie de leurs revenus : en 2014, 28 \% des migrants équatoriens en Espagne déclaraient avoir investi dans une maison en Équateur (Iglesias Martínez, 2015 : 110 ${ }^{10}$ ), un chiffre élaboré auprès des Équatoriens demeurés en Espagne et qui n'inclut donc pas ceux retournés au pays, alors même qu'on peut imaginer qu'une forte proportion d'entre eux y était propriétaires.

Avant de présenter plus finement certaines caractéristiques des maisons de retour observées lors de l'enquête de terrain, quelques réflexions peuvent être avancées sur les liens entre maisons et retour. On peut poser comme hypothèse principale qu'avoir construit une maison dans son pays d'origine est un élément facilitant la mise en œuvre du projet de retour. D'abord, le fait même d'avoir investi dans la maison dénote une identification durable au pays d'origine et la projection dans un projet de retour. Ensuite, on peut supposer que l'intérêt pour une maison au pays s'accompagne d'autres formes d'investissements matériels et immatériels transnationaux (maintien des liens avec la famille et les concitoyens, épargne, etc.) qui œuvreront aussi à la réalisation du retour. En outre, savoir que l'on aura un toit sur la tête semble de nature à encourager un projet de retour au pays si la situation dans le pays d'installation n'est plus satisfaisante. Les faits nous amènent pourtant immédiatement à nuancer cette hypothèse : les maisons des migrants restées vides, un trope de la littérature sur le retour, ne nous rappellent que trop bien qu'il ne suffit pas d'avoir bâti une maison en prévision de la retraite pour retourner y vivre. De quelle manière le logement fait-il ressource et est-il mobilisé (ou pas) lors de la mise en œuvre du retour?

\section{De la maison de retour à la maison des devises : des représentations à réactualiser dans le contexte urbain}

Dans une littérature que l'on qualifiera de « classique » sur les préparatifs du retour, souvent tournée vers le monde rural ou de petites villes (Boccagni, 2014 ; Calderón Bony, 2010 ; López, 2010 ; Massey et al., 2006 ; Pinson, 1999 ; Tesăr, 2018), les maisons de retour sont ostentatoires, singulières. Tranchant avec la modestie des demeures voisines, elles remplissent la double fonction de signifier le succès du migrant et de marquer son appartenance renouvelée à sa communauté d'origine. Constamment en travaux, elles réactivent aussi les liens avec les membres de la famille restés sur place, chargés de superviser la construction de la maison, de l'entretenir ou de l'occuper (Boccagni, 2014). L'enquête menée en milieu urbain, à Quito, a donné à voir d'autres facettes de la maison de retour. En écho aux observations sur les restructurations des métropoles latino-américaines et les effets urbains de l'expérience migratoire euro-

\footnotetext{
${ }^{10}$ D'après la même enquête, 35 \% des migrants équatoriens en Espagne possèdent leur propre maison en Équateur. Contrairement au chiffre précédent (28\%), celui-ci inclut les biens hérités (Ibid.).
} 
péenne développées dans d'autres contextes (Dureau et al., 2014), elle confirme l'importance de distinguer les territoires (ruraux, urbains, périurbains, à grands traits) et les types de quartiers, en raison des variations induites sur le marché résidentiel, et sur les stratégies des acteurs.

Le choix de bâtir sa maison en ville est le fait de migrants originaires de la ville en question, mais pas seulement : la plupart des enquêtés, quiteños, situent leur maison dans la capitale, souvent dans un quartier qui n'est pas celui dans lequel ils résidaient au moment de leur départ ; d'autres ont acheté des biens dans des villes où ils n'avaient pas vécu avant de partir en Espagne. Contrairement au réancrage dans le lieu d'origine que signifiait la maison du retour dans la littérature classique, la localisation obéit donc à d'autres logiques, incluant des lieux-tiers, comme cela a été également observé dans le cadre des migrations sénégalaises (Lessault, 2014).

Par ailleurs, en ville, les « maisons » des migrants sont souvent des appartements. La dimension ostentatoire de la maison des devises s'efface alors, ainsi que son caractère perpétuellement inachevé. À noter cependant que des appartements peuvent se situer dans des maisons dont les proportions les désignent immédiatement comme appartenant aux migrants. Ainsi, dans le quartier populaire de Llano Grande, à la périphérie de Quito, le marché foncier a permis aux migrants d'hériter ou d'acquérir de grands lots, sur lesquels construire des maisons aux proportions imposantes, dont certaines sont divisées en plusieurs appartements. La finalité locative est, peut-être, la caractéristique la plus notable des maisons des migrants en ville. Aucune maison vide parmi les biens de mes enquêtés en Équateur : toutes étaient louées et procuraient à leurs propriétaires des revenus réguliers (plusieurs centaines d'euros par mois). La localisation en ville est donc en soi une ressource, dont avaient conscience les migrants au moment de l'achat. Le pari fait sur la rentabilité de ces investissements locatifs est conforté par une frénésie économique et discursive favorable au développement immobilier, dans laquelle ont baigné les migrants en Espagne jusqu'en 2008, puis en Équateur (Quentin, 2016).

Car si le rêve de construire une maison est au centre de nombreux projets migratoires, il est aussi façonné par des acteurs, publics et privés, bien identifiables en milieu urbain. Le gouvernement Correa, très actif en faveur des migrants, a ainsi mis en place un «bono de la vivienda » (" subvention au logement »), programme d'incitation à l'achat de maisons dans des lotissements définis, situés dans les périphéries des principales villes équatoriennes, en accord avec des banques qui fournissaient les crédits aux migrants, et des promoteurs immobiliers qui édifiaient les biens. Cette offre publique, en vigueur jusqu'en 2015 au moins ${ }^{11}$, était encadrée par certaines conditions (localisation, obligation de fournir un apport de plusieurs milliers de dollars pour pouvoir bénéficier de la subvention et du crédit, interdiction de revendre le bien avant cinq ans). En parallèle se développe une offre privée, diffusée par des agences immobilières associées

\footnotetext{
${ }^{11}$ Ce programme apparaît dans les rapports du ministère du Logement équatorien jusqu'en 2015. La politique migratoire équatorienne s'infléchit ensuite pour des raisons très diverses (fin de la présidence de Correa, tremblement de terre en 2016, afflux de populations nouvelles, comme les Vénézuéliens, les Haïtiens, les Colombiens).
} 
à des promoteurs qui ciblent les migrants à l'étranger et les incitent à investir dans les villes de leur pays d'origine. Ecuaviviendas en est un bon exemple. Très active sur internet (Facebook, WhatsApp) mais disposant d'un bureau à Madrid, cette agence immobilière propose aux Équatoriens en Espagne des maisons individuelles toutes identiques, dans des lotissements périphériques de villes équatoriennes, ainsi que des crédits pour les financer. Une phrase revient sur chaque publication : "La meilleure façon d'assurer ton argent, c'est d'investir dans ton futur, car la plus-value est très élevée, tu envisages un nouveau départ dans ta vie, avec ta famille ${ }^{12}$. "Ecuaviviendas associe la maison des migrants à la sécurité, à la famille, au renouveau, mais aussi à un investissement économique : la maison est vendue comme un placement financier fortement teinté d'affect. L'intérêt de ce type d'acteurs pour les maisons des migrants, leurs discours entretenant des sentiments nostalgiques peuvent être mis en relation avec le développement, dans les pays d'origine des migrants, de politiques visant à capter les rentes migratoires. Après avoir canalisé les devises vers le développement agricole et productif (Rebaï, 2014), l'État équatorien semble soucieux de mettre aussi à profit ces devises dans le développement urbain. Quant aux promoteurs et agences, ils forment un « marché transnational de l’immobilier », également observé en Inde (Varrel, 2014). Si une majorité des migrants que j'ai rencontrés avaient financé leurs biens par leur épargne, quelques-uns avaient eu recours à ces acteurs privés et à un financement par le crédit bancaire ; la rencontre s'était faite au cours des salons de l'immobilier très en vogue en Espagne pendant le boom des années 2000.

Il faut noter, enfin, que l'investissement immobilier des migrants équatoriens ne s'est pas limité à leur pays d'origine. Alors que leurs revenus les classaient parmi les ménages modestes, 35,2 \% des Équatoriens en Espagne y étaient propriétaires de leur logement (Iglesias, 2015 : 88), grâce aux politiques migratoires espagnoles ayant facilité l'accès aux titres de séjour et à la citoyenneté pour les Latino-Américains, et aux conditions d'accès au crédit très favorables - mais très fragiles - en vigueur avant l'effondrement du système économique espagnol en 2008. Ce patrimoine multisitué est révélateur de vies en tension entre plusieurs espaces (Boccagni, 2014). De fait, à l'exception de Lupe et de son mari, qui ont choisi de rester locataires en Espagne pendant vingt ans et ont préféré réaliser deux acquisitions à Quito, et de Julia, qui s'est méfiée du système bancaire et n'a investi nulle part, tous les enquêtés avaient acheté un logement en Espagne. Même si elles doivent encore être étayées, les premières données de terrain indiquent par ailleurs que ceux arrivés en Espagne au début des années 2000, c'est-àdire au moment où l'appel à investir dans l'immobilier était le plus fort, ont repoussé le financement de la maison en Équateur pour privilégier le remboursement du crédit en Espagne. Pour autant, l'achat en Espagne n'excluait pas à leurs yeux un éventuel

\footnotetext{
12 « La mejor forma de asegurar tu dinero, es invertir en tu futuro ya que la plusvalía es muy alta, y piensas en nuevos aires en tu vida y junto a la familia », en ligne : https://www.facebook.com/ EcuaviviendaS-408704035984477/ (extrait publication du 11 mars 2020 sur la page Facebook de l'agence).
} 
retour en Équateur ${ }^{13}$. En effet, le discours en vigueur à l'époque, diffusé par les acteurs de l'immobilier, repris par les médias, intégré par les citoyens ordinaires, professait une hausse incontestable du marché immobilier. La possibilité de revente avec profit était posée comme une évidence, si bien que paradoxalement, acheter en Espagne paraissait une opération financière sûre, compatible avec un projet de retour : il suffirait de revendre le bien et de s'établir en Équateur avec la plus-value. Acheter en Espagne est donc à la fois un choix résidentiel à court terme et un investissement à long terme.

$\mathrm{Au}$ moins autant qu'une logique ostentatoire, identitaire ou affective, souvent attachée aux maisons des migrants, la logique économique d'investissement transnational est donc déterminante dans le contexte urbain. La localisation des biens, leur fonctionnalité, mais aussi leur morphologie, qui dans la plupart des cas se fond dans le tissu urbain, dessinent d'autres fonctions à la « maison des devises » que celles mises en avant dans une littérature classique établie à partir de territoires peu valorisés sur le marché résidentiel. Bien avant de loger leurs propriétaires, ces biens immobiliers jouent un rôle non négligeable dans l'économie quotidienne des ménages, en fournissant une source de revenus qui varie selon les villes et les quartiers, mais qui s'élevait à plusieurs centaines de dollars mensuels pour la majorité de mes enquêtés. L'argent circule donc du nord au sud (épargne et investissement) mais aussi du sud au nord (rente locative), selon la logique des « devises inversées » (Mazzucato, 2010). Comment opère ce capital dans les projets de retour?

\section{Une maison pour le retour ? Les scénarios initiaux mis à l'épreuve par la crise}

En déstabilisant les positions économiques, la crise de 2008 en Espagne a bousculé la temporalité du retour pour de nombreux ménages. Comment le patrimoine immobilier pèse-t-il dans l'alternative qui s'offre à de nombreux ménages entre partir ou rester ? Peu de données permettent d'établir combien de personnes sont rentrées, dans quelles zones d'Équateur, et dans quelle mesure elles avaient préparé leur réinstallation, d'autant moins que les retours se sont faits à distance des programmes mis en place par l'État équatorien (Parella, Petroff, 2014). En l'absence de ce cadrage quantitatif, l'enquête qualitative révèle plusieurs scénarios dans lesquels les investissements immobiliers influent sur les mobilités, et ce pas toujours dans le sens qu'on aurait pu attendre, à savoir une corrélation entre le fait de détenir une maison dans le pays d'origine et une plus grande propension au retour.

Plusieurs tendances peuvent être distinguées pour des migrants ayant acheté une maison à Quito, avec des variations autour de la réalisation ou non du retour : le retour avec relogement dans la maison des devises (1.a) ; le retour au prix de la revente de la maison (1.b) ; le maintien en Espagne, vécu comme temporaire (2.a) ou définitif (2.b), grâce aux ressources locatives en Équateur.

\footnotetext{
${ }^{13}$ Entretiens à Madrid, 2018.
} 
Dans la première configuration, le retour, préparé de longue date, a été mis en œuvre. Ceux pour qui la réinstallation s'est le mieux passée (1.a), selon leur propre évaluation, étaient propriétaires non pas d'un mais de plusieurs biens à Quito, un qu'ils ont occupé, et un ou plusieurs autres qui leur ont fourni un revenu avant qu'ils parviennent à se réinsérer sur le marché de l'emploi, une étape difficile pour beaucoup de migrants de retour. Fait notable, ce capital immobilier composé de biens multiples est souvent constitué à partir d'un héritage familial, un terrain ou une maison, revendu en vue d'un nouvel achat dans un quartier plus attractif. La mise de fonds initiale est complétée par l'épargne constituée par les migrants, avec l'aide de leurs proches (parents, frères et sœurs), qu'ils aient migré ou non. Diana est l'exemple de cette mutualisation fructueuse des ressources familiales ${ }^{14}$. De 1993 à 2011, elle a passé 18 ans en Espagne, où elle a travaillé comme employée domestique, livreuse, serveuse. Avec ses deux sœurs, également établies à Madrid, elles ont vendu un terrain agricole hérité de leur grandmère à l'extérieur de Quito pour acheter, en ajoutant leur épargne, une parcelle dans un quartier plus central de la ville. Elles y ont fait construire un immeuble de vingtquatre appartements et deux locaux commerciaux. Chaque sœur est propriétaire de huit appartements. Pendant son séjour en Espagne, Diana en louait deux, et faisait des travaux progressivement dans les six autres. Depuis son retour à Quito, elle en occupe un et loue les sept autres. Elle en tire un revenu de $2000 \$ /$ mois, une somme conséquente en Équateur. Cette gestion conjointe des ressources éclaire de façon originale l'importance, par ailleurs bien établie, de l'entraide familiale dans les projets migratoires. Elle est révélatrice de l'implication à la fois des individus mobiles et immobiles dans la préparation des projets de retour (Cortés, Oso, 2017), et constitue une variante d'autres formes de coopération familiale autour de la maison, observées en milieu rural (supervision des travaux par des proches, occupation de la maison par des membres de la famille) (Boccagni, 2013 ; Pinson, 1999; Tesăr, 2018).

Pour ceux qui ne possédaient qu'un bien, le retour est plus difficile. À son arrivée en Équateur, Alberto a même dû revendre la maison qu'il y avait achetée et s'installer avec femme et enfants chez ses beaux-parents ${ }^{15}$. L'argent tiré de la vente lui a permis de financer les billets d'avion Madrid-Quito et de dégager de l'épargne, le temps de consolider sa position sur le marché de l'emploi (1.b).

Mais le retour n'est pas toujours possible. Les situations où, bien que préparé de longue date, il n'a pas lieu, constituent un second cas de figure. De nombreux éléments soulevés dans les entretiens amènent à repousser le retour (2.a) : absence de perspective d'emploi dans le pays d'origine, refus des enfants de rentrer, mais aussi goût pour la vie en Espagne. La dette immobilière contractée en Espagne peut être un facteur de mise en suspens des projets de mobilité. Ainsi Gladys, une soixantaine d'années ${ }^{16}$, a vu sa situation se dégrader drastiquement après 2008: le taxiphone dont elle était propriétaire avec son mari s'est vidé. Le couple est passé de l'aisance économique à une quasi-ab-

\footnotetext{
${ }^{14}$ Entretien à Quito, 2015.

15 Entretien à Quito, 2015.

16 Entretien à Madrid, 2018.
} 
sence de revenus et à une retraite forcée. Sa fille et ses petits-enfants sont retournés vivre à Santo Domingo, une des principales villes équatoriennes. Gladys y possède une maison qu'elle a terminé de payer. Mais elle est aussi, selon ses termes, «propriétaire d'une dette en Espagne ", suite à l'achat d'un appartement avec un crédit qu'elle ne peut plus honorer mais que la banque refuse d'effacer. Or le nom de son fils figure sur le contrat bancaire. Beaucoup d'histoires ont circulé, en Équateur et en Espagne, à propos de migrants endettés en Espagne, rentrant du jour au lendemain selon le scénario de "fuite » mentionné plus haut, et poursuivis par les banques jusque dans leur pays d'origine ${ }^{17}$. Si l'idée de rentrer a traversé l'esprit de Gladys, comment l'exécuter, avec l'angoisse que la dette ne poursuive son fils et ne l'empêche de construire sa vie ? En espérant qu'une solution judiciaire finira par être trouvée grâce à l'important mouvement social de contestation de la dette, qu'elle a rejoint comme de nombreux Équatoriens (Ravelli, 2018 ; Suárez, 2017), elle attend à Madrid, dans son appartement menacé de saisie. Les quelques centaines d'euros de la rente que lui rapporte sa maison en Équateur sont l'un de ses principaux revenus.

Autre variation, le maintien en Espagne devenu projet définitif, grâce aux possibilités financières offertes par les biens possédés en Équateur (2.b). Luis, 70 ans ${ }^{18}$, a toujours projeté de rentrer en Équateur. D’origine très modeste, avec la coopération financière de ses enfants, également installés en Espagne, il a pris soin d'acheter deux maisons dans la périphérie de Quito, dont l'une est subdivisée en trois appartements, tous loués. De même que ses enfants, il est aussi propriétaire de l'appartement qu'il occupe en Espagne. Aujourd'hui à la retraite après une vie de labeur, Luis réalise que ses enfants se plaisent en Espagne, et que s'il doit rentrer, ce sera seul. L'histoire, classique, devient intéressante lorsque l'on comprend que le renoncement au retour, d'abord subi, est devenu accepté le jour où Luis et ses enfants ont acquis, ensemble, une maison secondaire dans un village à une heure de Madrid. Luis y passe ses semaines, y cultive un potager et se voit y vieillir. Cette maison, ainsi que l'ensemble du capital immobilier détenu par la famille en Espagne et en Équateur, a été financée selon le modèle de mutualisation des ressources mis en évidence plus haut : crédit bancaire et association de l'épargne de Luis, de son épouse et de leurs deux enfants, alors même que tous occupent des postes modestes (aide à la personne pour les femmes, manutention et bâtiment pour les hommes). Grâce au revenu constant du capital immobilier progressivement constitué à Quito et mis en location, la famille a conservé une petite capacité d'épargne. Elle s'est avérée décisive pour traverser la crise économique sans trop de heurts, malgré des périodes de chômage, en honorant les paiements des appartements madrilènes, et au moment d'acheter en Espagne la maison du «non-retour ».

\footnotetext{
$\overline{17}$ Le gouvernement Correa a pris des mesures légales pour empêcher les banques de prélever des hypothèques sur des biens détenus en Equateur par des migrants endettés en Espagne, sans parvenir à rassurer complètement la population. Voir par exemple l'article « El largo brazo de los bancos no llega a Ecuador », El Pays, mis en ligne le 13 novembre 2012. URL : https://elpais.com/ politica/2012/11/13/actualidad/1352831394_832868.html.

18 Entretien à Madrid, 2018.
} 
Plus largement, on constate que les ménages qui ne peuvent rentrer en Équateur ou souhaitent rester en Espagne développent des stratégies transnationales de mobilité des capitaux économiques constitués par l'immobilier : mise en location d'un appartement en Équateur pour compenser les revenus en baisse en Espagne ; vente d'un terrain pour tenter de rembourser la dette ou pour débloquer une épargne servant d'apport pour louer un logement en Espagne. Dans ce cas, la propriété joue un rôle de filet de sécurité. Mais elle opère de façon très imparfaite et souvent frustrante pour les ménages : en raison du différentiel entre les économies espagnoles et équatoriennes, la vente d'un terrain en Équateur ne constitue pas toujours le bénéfice escompté. Le capital immobilier accumulé se transfère donc mal d'un contexte à l'autre. Il permet toutefois d'atténuer l'incertitude économique et la précarité auxquelles sont confrontés les migrants, plus encore depuis 2008.

Posséder une maison dans le pays d'origine n'est donc pas un déterminant pour les projets de retour, même si cela peut les faciliter. Plus que comme un logement, la maison du retour joue surtout un rôle conséquent comme capital économique, susceptible d'être valorisé, revendu, converti en épargne, transféré. Elle peut alors soit être mise à profit pour le retour, en libérant une rente facilitant la réinstallation ; soit, paradoxalement, permettre d'éviter le retour, que ce soit pour rester en Espagne ou - une configuration encore peu explorée - envisager des mobilités vers de nouvelles destinations (Palash, Baby-Collin, 2019).

\section{Conclusion}

Loin de l'image de la maison disproportionnée mais vide, évoquée dans la littérature classique sur les préparatifs du retour, et souvent analysée sous l'angle du rapport au lieu d'origine, les maisons des migrants observées dans les villes équatoriennes relèvent de logiques économiques privilégiant la constitution d'un capital transnational. À ce titre, elles sont des «maisons des devises », bien plus que des maisons de retour. À l'instar d'autres ressources a priori conçues comme donnant lieu à des ancrages (titres de séjour, citoyenneté espagnole), elles opèrent en outre plutôt comme des leviers de mobilité géographique. Pour autant, on n'observe pas de relations de causalité simples. Paradoxalement, des appartements achetés en Espagne ont été envisagés au moment de l'acquisition comme une ressource permettant à moyen terme de retourner au pays, tandis que des maisons bâties dans le pays d'origine servent à financer le séjour en Espagne.

Ces résultats amènent à relire la signification généralement donnée à la propriété, notamment pour les migrants pour lesquels l'achat, que ce soit dans le pays d'origine ou de destination, tend à être interprété comme marqueur d'appartenance ou comme gage de sédentarisation. Sans que ces significations disparaissent, elles se doublent d'une conception plus pragmatique du capital immobilier. Les maisons, outre leurs dimensions affectives, identitaires, sont appréhendées comme des ressources cumulables, transférables. On observe ainsi chez de nombreux Équatoriens aux revenus modestes 
en Espagne des stratégies de constitution d'un patrimoine immobilier multisitué. Elles se composent souvent à l'échelle des familles, et non seulement des ménages : elles reposent sur la mise en commun de ressources, sur leur conversion et leur circulation d'un espace à l'autre, ce qui rend le cadre théorique de «l'accumulation transnationale de capitaux » très pertinent sur le plan théorique pour penser le phénomène.

Cette lecture permet d'éclairer des dynamiques jusqu'ici peu visibles dans la littérature sur la «maison des devises », en mettant en évidence comment l'investissement dans ces maisons rencontre des logiques de marché immobilier développées dans les villes du Sud, stimulées par des acteurs opérant à l'échelle nationale et parfois globale (États, promoteurs et agences immobilières). Ce point demande à être approfondi, mais représente une piste de recherche qui enrichit les représentations sur les maisons du retour, en concordance avec les résultats d'autres travaux sur la question (Lessault, 2014 ; Varrel, 2014). Il permet également de voir que les migrants ont souvent investi dans des quartiers dans lesquels ils n'avaient pas résidé, et qu'ils ont choisis pour leur dynamisme résidentiel, anticipant les revenus que leur bien pourra leur apporter pendant leur migration.

Les migrants s'efforcent donc de réaliser des investissements économiques a priori rationnels, sécurisants, selon le discours néolibéral dominant qui valorise l'investissement dans la pierre. Pour autant, l'exemple des Équatoriens en Espagne en révèle la fragilité : les scénarios initiaux sont contrariés par la crise de 2008, qui peut tantôt précipiter les retours, tantôt les empêcher. Outre l'intérêt de comparer les comportements des Équatoriens avec ceux d'autres groupes migrants en Espagne, comme les Marocains, il serait intéressant de comprendre dans quelle mesure les stratégies, ici observées chez des ménages ayant réalisé ces investissements avant 2008, évoluent après la crise.

Échappant aux simplifications, l'articulation entre les mobilités et la construction de patrimoines immobiliers à l'échelle familiale, la circulation de ces capitaux et leur conversion d'un espace à l'autre, du nord au sud et du sud au nord, apparaît donc comme une piste de recherche stimulante pour comprendre la complexité des projets migratoires, la recomposition des inégalités dans l'espace transnational, et leurs traductions en trajectoires géographiques.

\section{Bibliographie}

Baby-Collin V., Cortes G. (2014), « Nuevos despliegues del campo migratorio boliviano frente a la crisis », Revista CIDOB d'Afers Internacionals, vol. 106-107, p. 61-83.

Boccagni P. (2014), "What's in a (Migrant) House? Changing Domestic Spaces, the Negotiation of Belonging and Home-making in Ecuadorian Migration », Housing, Theory and Society, vol. 31, n 3, p. 277-293

Calderón Bony F. (2010), "L'espace d'habitation comme miroir identitaire. Le cas

des migrants de Patamban (Michoacán, Mexique) aux États-Unis », Cahiers des Amériques latines, $\mathrm{n}^{\circ}$ 59, p. 57-78. 
Cerrutti M., Maguid A., Diaz Gil A. (2011), " Migrantes sudamericanos en España: panorama y políticas », Cuadernos migratorios_OMI, $\mathrm{n}^{\circ}$ 1, p. 314.

Colectivo I. (2011), « Notas sobre los efectos socioeconómicos de la crisis para la población inmigrada », Papeles de relaciones ecosociales y cambio global, $n^{\circ}$ 113, p. 85-95.

CoRTÉs A. (2011), « Retorno y Acumulación de Activos. Aproximaciones Etnográficas al caso de Ecuador y España », in J. GinIENIEWicz (dir.), La migración latinoamericana a España: una mirada desde el modelo de acumulación de Activos, Quito, Universidad de Manchester y FLACSO-Ecuador, p. 81-105.

Cortés A., Oso L. (2017), « Avecillas y pájaros en vuelo transnacional: Retorno, género y estrategias de movilidad e inmovilidad entre Ecuador y España », Revista Española de Socioogía, vol. 26, n 3, DOI: 10.22325/fes/res.2017.28.

Dureau F., Lulle T., Souchaud S., Contreras Y. (2014), Mobilités et changement urbain : Bogotá, Santiago et São Paulo, Rennes, Presses universitaires de Rennes.

EsPinosa V. M. (1998), El dilema del retorno: migración, género y pertenencia en un contexto transnacional, Zapopan, Jal./Zamora, Michoacán, México, El Colegio de Jalisco/El Colegio de Michoacán.

Glick Schiller N. (1999), "Transmigrants and Nation-States: Something Old and Something New in the U.S. Immigrant Experience », in C.H. Hirschmann, P. Kasinitz, J. De Wind (dir.), The Handbook of International Migration, New York, Russell Sage Foundation, p. 94-119.

GUARNIZO L. (2014), Emigración internacional y retorno en la región andina, communication présentée à SEPMIG, El Colegio de la Frontera Norte, en ligne. URL : http://www. youtube.com/watch?v=BWJwgMlxcqw.

Herrera G., Pérez Martínez L. (2015), « ¿Tiempos de crisis, tiempos de retorno? Trayectorias migratorias, laborales y sociales de migrantes retornados en Ecuador », Estudios Políticos, $\mathrm{n}^{\circ}$ 47, p. 221-241.

Iglesias Martínez J. (2015), La población de origen ecuatoriano en España: características, necesidades y expectativas en tiempos de crisis (Primera edición), Madrid, Embajada del Ecuador en España.

Koller S. (2010), «Équateur : la politique des droits », Problèmes d’Amérique latine, vol. 75, $\mathrm{n}^{\circ} 1, \mathrm{p} .61-73$.

Lessault D. (2014), « Des espaces parcourus aux lieux investis. Enquête auprès des Sénégalais installés en Europe », Autrepart, vol. 67-68, n 4, p. 213-232.

Larramona G. (2013), « Espagne : l'émigration des immigrés », Population, vol. 68, n² 2 , p. 249-271.

LÓPEZ DE LERA D. (2010), « Emigración, inmigración y retorno: tres etapas de un mismo proceso », Polígonos, n² 20, p. 9-27. 
López S. L. (2010), « The Remittance House: Architecture of Migration in Rural Mexico », Buildings er Landscapes: Journal of the Vernacular Architecture Forum, vol. 17, $\mathrm{n}^{\circ} 2$, p. 33-52.

Ma Mung E. (2009), " Le point de vue de l'autonomie dans l'étude des migrations internationales : "penser de l'intérieur" les phénomènes de mobilité », in F. DUREAU, M.-A. Hily (dir.), Les mondes de la mobilité, Rennes, Presses universitaires de Rennes, p. 25-38.

MarTínez Buján R. (2016), « La experiencia del retorno de los migrantes bolivianos desde España: la toma de la decisión y la reinserción en origen desde una perspectiva de género », Amérique latine Histoire et Mémoire. Les Cahiers ALHIM, n 31, juin. En ligne. URL : http://alhim.revues.org/5506.

Martiniello M, ReA A. (2011), "Des flux migratoires aux carrières migratoires », SociologieS. En ligne. URL : http://sociologies.revues.org/3694.

MazzucATo V. (2010), « Reverse remittances in the migration-development nexus: twoway flows between Ghana and the Netherlands », Population, Space and Place, vol. $17, \mathrm{n}^{\circ} 5, \mathrm{p} .454-468$.

Moreno-Márquez G, Álvarez-Román J. (2017), " Evidencias empíricas sobre el retorno migratorio España-Ecuador. ¿Discurso, mito o realidad? », Papeles de Población, vol. 23, nº 91 , p. s/p.

Palash P, Baby-Collin V. (2019), « The other side of need: Reverse economic flows ensuring migrants' transnational social protection », Population, Space and Place, vol. 25, n 5 , https://doi.org/10.1002/psp.2219 .

Parella S, Petroff A. (2014), « Migración de retorno en España: salidas de inmigrantes y programas de retorno en un contexto de crisis », Anuario CIDOB de la Inmigración, Barcelone, CIDOB, p. 63-87.

Perraudin A. (2016), « Crise économique et migrations de retour. Le cas des Équatoriens en Espagne », Autrepart, vol. 77, n 1, p. 165-182.

Pinson D. (1999), «Les maisons du marocain », in P. BONNIN, R. de VILLANOVA (dir.), D'une maison l'autre : parcours et mobilités résidentielles, Grane, Créaphis Éditions, p. 69-88.

Quentin A. (2016), « La politique du logement en Équateur : de la bonne gouvernance au Buen Vivir, un tournant postnéolibéral ? », Cahiers des Amériques latines, n 83, p. 53-73.

Ramos C. (2017), " Onward migration from Spain to London in times of crisis: the importance of life-course junctures in secondary migrations », Journal of Ethnic and Migration Studies, vol. 44, n 11, p. 1-17.

Ravelli Q. (2018), " Peut-on parler d'un mouvement postcolonial en Espagne ?», Mouvements, $\mathrm{n}^{\circ}$ 94, p. 126-140. 
RebAï N. (2014), «Quand l'argent de la migration change la donne : développement agricole et dynamique foncière dans une localité de la province andine de l'Azuay (Équateur) », Autrepart, vol. 67-68, n 4, p. 193-212.

SAYAD A. (1975), « Elghorba : le mécanisme de reproduction de l'émigration », Actes de la recherche en sciences sociales, vol. $1, \mathrm{n}^{\circ} 2$, p. 50-66.

Schramm C. (2011), « Retorno y Reinserción de Migrantes Ecuatorianos. La Importancia de las Redes Sociales Transnacionales », Revista CIDOB d'Afers Internacionals, vol. 9394, p. 241-260.

SuÁrez M. (2017), « Debt revolts: Ecuadorian foreclosed families at the PAH in Barcelona », Dialectical Anthropology, vol. 41, n 3, p. 263-277.

SuÁreZ-GRimalt L. (2017), « Migración y movilidad social: una aproximación desde las estrategias de acumulación de activos de la población latinoamericana en España », Revista Española de Sociología, vol. 26, nº 3, p. 345-368

TARRIUS A. (2001), «Au-delà des États-nations : des sociétés de migrants », Revue européenne des migrations internationales, vol. 17, $\mathrm{n}^{\circ} 2$, p. 37-61.

Tesăr C. (2018), « Houses under Construction », in M. Brazzabeni, M.I. Cunha, M. FotTA (dir.), Gypsy Economy: romani livelihoods and notions of worth in the 21st century, New York, Berghahn Books, p. 181-200.

VARREL A. (2014), " L'émergence d'un marché transnational de l'immobilier indien », Autrepart, vol. 67-68, n² 4, p. 233-246.

VEGA C. (2016), « Complejizando el "retorno productivo". Mujeres y hombres ante el regreso a Ecuador desde España », Amérique latine Histoire et Mémoire. Les Cahiers ALHIM, $\mathrm{n}^{\circ}$ 31, juin, en ligne. URL : http://alhim.revues.org/5498. 